\title{
Perspectivas para un estudio sobre bilingüismo en universidades regionales colombianas ${ }^{1}$
}

\author{
Perspectives for a study on bilingualism in Colombian regional universities \\ Perspectivas para um estudo sobre bilinguismo em universidades regionais \\ colombianas
}

\author{
Nélida Cárdenas Vergaño ${ }^{2}$ \\ Corporación Universitaria Iberoamericana, Colombia \\ Grupo de Investigación HISULA
}

Recepción: 05/07/2017

Evaluación: 26/02/2018

Aceptación: 29/06/2018

Artículo de Investigación

DOI: $10.19053 / 01227238.8566$

\section{RESUMEN}

En el presente artículo se hace una reflexión sobre las tendencias del bilingüismo en Colombia, brindando una reseña histórica de la forma como se introdujo al país la enseñanza de los idiomas extranjeros, además de abordar algunos aspectos conceptuales y teóricos sobre la definición $\mathrm{y}$ tipos de bilingüismo que han marcado influencia para las propuestas del Ministerio de Educación Nacional en el área. Más adelante se describe el Proyecto de Fortalecimiento a Licenciaturas en Idiomas/Inglés, propuesto en el marco del Programa Nacional de Bilingüismo-PNB 2004-2019, todo ello con el fin de presen- tar los fundamentos metodológicos de la propuesta investigativa "Políticas bilingües y su impacto en la identidad cultural y calidad educativa en programas de licenciatura en lenguas - inglés en Colombia, 2004-2014", correspondiente al Doctorado en Ciencias de la Educación de Rudecolombia - CADE, Tunja, grupo de investigación Historia y Prospectiva de la Educación en América Latina - HISUlA.

Palabras clave: Revista Historia de la Educación Latinoamericana; bilingüismo; educación superior, política educativa.

1 Artículo de investigación. Construcción de la tesis doctoral "Políticas bilingües y su impacto en la identidad cultural y calidad educativa en programas de licenciatura en lenguas - inglés en Colombia, 2004-2014", realizada desde el Doctorado en Ciencias de la Educación de Rudecolombia - CADE, Tunja, adscrita al Grupo de Investigación Hisula. Directora de tesis: Dra. Doris Lilia Torres.

2 Magistra en Educación. Estudiante del Doctorado en Ciencias de la Educación de Rudecolombia - Cade, Tunja. Docente de la Corporación Universitaria Iberoamericana - Colombia. Miembro del Grupo de Investigación Historia y Prospectiva de la Educación en América Latina - HISUlA. Correo electronico: teachernelida@gmail.com 


\section{ABSTRACT}

This article reflects on the trends of bilingualism in Colombia by providing a historical overview of how the teaching of foreign languages was introduced to the country. There are also analyzed some conceptual and theoretical aspects about the definition and types of bilingualism that have influenced the proposals of the Ministry of National Education in this domain. Secondly, we describe the "Project to Strengthen Bachelor's Degrees in Languages / English", proposed within the framework of the National Program of Bilingualism-PNB 2004-2019, in order to present the methodological foundations of the research proposal "Bilingual policies and their impact on the cultural identity and educational quality in undergraduate programs in languages - English- in Colombia, 2004-2014 ". This Project is registered in the Doctorate in Educational Sciences - Rudecolombia - CADE, Tunja, Research group History and Prospective studies Latin American Education - HISULA.

Keywords: Journal History of Latin American Education; bilingualism; higher education, education policy.

\section{RESUMO}

O presente artigo apresenta uma reflexão sobre as tendências do bilinguismo na Colômbia, fornecendo uma visão histórica da forma como o ensino de idiomas estrangeiros, além de abordar alguns aspectos conceituais e teóricos sobre a definição e tipos de bilinguismo que influenciaram as propostas do Ministério da Educação Nacional na área. Mais adiante se descreve o Projeto de Fortalecimento a Licenciaturas em Idiomas/Inglês, proposto no marco do Programa Nacional de Bilinguismo-PNB 2004-2019, tudo isso com o fim de apresentar os fundamentos metodológicos da proposta investigativa "Políticas bilíngues e seu impacto na identidade cultural e qualidade educativa nos programas de licenciatura em línguas - inglês na Colômbia, 2004-2014", correspondente ao Doutorado em Ciências da Educação de RUDECOLOMBIA - CADE, Tunja, grupo de pesquisa História e Prospectiva da Educação na América Latina HISULA.

Palavras-chave: Revista História da Educação Latino-americana; bilinguismo; educação superior, política educativa.

\section{INTRODUCCIÓN}

En las últimas décadas, y gracias a "la importancia asignada a la adquisición de una segunda lengua como garantía para la competitividad en un mundo cada vez más globalizado"3 e implementando varios programas y proyectos de notable influencia en los currículos de los distintos niveles educativos colombianos; en ese sentido también se han venido realizando investigaciones y trabajos teóricos que ayudan a fundamentar conceptos, metodologías de enseñanza-aprendizaje de las lenguas extranjeras y herramientas a utilizar para la implementación de esos programas y proyectos en las instituciones educativas del país. Con el presente artículo de reflexión se pretende profundizar sobre las tendencias, normatividades, referentes conceptuales y perspectivas teóricas relacionadas con esa temática.

3 Jenny Raquel Bermúdez y Yamith José Fandiño, "El fenómeno bilingüe: perspectivas y tendencias en bilingüismo", Revista de la Universidad de La Salle, n. ${ }^{\circ} 59$ (2012): 99. 
En la primera parte se desarrolla una breve reseña histórica de la manera como se fue introduciendo la enseñanza de los idiomas extranjeros en la educación colombiana, además de realizar un recorrido por las principales normatividades institucionales alrededor del área de bilingüismo. En un segundo momento se asumen aspectos conceptuales y teóricos sobre la definición y tipos de bilingüismo, analizando su influencia en la propuesta bilingüe nacional. En tercer lugar se describe el Proyecto de Fortalecimiento a Licenciaturas en Idiomas/ Inglés, propuesto en el marco del Programa Nacional de Bilingüismo (PNB) 20042019, además de dar a conocer las tendencias reflexivas sobre el PNB por parte de académicos y estudiosos del área. Finalmente se presentan las generalidades de la propuesta investigativa doctoral "Políticas bilingües y su impacto en la identidad cultural y calidad educativa en programas de licenciatura en lenguas - inglés en Colombia, 2004-2014". Las siguientes líneas corresponden a la fase preliminar del proyecto de investigación, por lo que se seguirá profundizando en temas también pertinentes a esta propuesta, tales como políticas y calidad educativa, currículo, educación superior regional, cultura e identidad y, por supuesto, el mismo bilingüismo.

\section{Exploración histórica del bilingüismo en Colombia}

Desde la perspectiva de Aline $\mathrm{Helg}^{4}$, en la época de la Colonia se inició en Colombia la implantación del español como lengua de instrucción para la enseñanza del cristianismo y como dominio cultural y político, además de la introducción del latín y el griego como lenguas de acceso a la academia. En el periodo de la Independencia dominaban en Colombia las influencias culturales inglesa y francesa, que marcaron derroteros en la enseñanza del inglés y el francés, al tiempo que perdía dominio la enseñanza y el uso del latín en las instituciones escolares. Hacia 1870, en el plano educativo comienzan a fortalecerse en Colombia las ideas pedagógicas de Pestalozzi, Froebel y Herbart, que dominaron las propuestas escolares de la época; bajo esa perspectiva llegó una misión de educadores alemanes a dirigir la formación pedagógica de las escuelas normales, pero tuvo dificultades debido a su limitado dominio del español y a las diferencias religiosas y políticas que se daban en el país por esa época, debiendo terminar esa propuesta ocho años después.

Acerca del inicio de la enseñanza de los idiomas en Colombia, Jaime Usma Wilches ${ }^{5}$ afirma que

Language policies and reforms agendas preceding the National Program in Colombia can be traced to the times of the colony. After the colonization of the "new" continent, Catholic missionaries were effective in imposing their languages, main-

4 Aline Helg, La educación en Colombia, 1918-1957: Una historia social, económica y política, 2. ${ }^{a}$ ed. (Bogotá: Universidad Pedagógica Nacional/Plaza y Janés Editores, 2001), 22.

5 Jaime A. Usma Wilches, "Education and Language Policy in Colombia: Exploring Processes of Inclusion, Exclusion, and Stratification in Times of Global Reform", Profile: Issues in Teachers' Professional Development, 11, n. ${ }^{\circ} 1$ (2009): 2. 
ly Spanish, Greek, and Latin. Later on, after the independence of the region from Spain two centuries ago, the new ruling elite started to send their children to Europe, which then led towards the importing of books and ideas associated with languages such French, German, and English. These moves paved the road to these languages into the country and their association with enlightened ideas and intellectual elites, while indigenous and Creole languages started to be associated with ignorance and undervelopment ${ }^{6}$

A partir de la Constitución de 1886 se modificó la división política y administrativa de Colombia, lo que se reflejó en cambios importantes en el ámbito educativo; se hizo énfasis en la alfabetización en español y la enseñanza de los idiomas extranjeros pasó al segundo plano ${ }^{7}$. Con la creación de la Facultad de Educación de Tunja, bajo el decreto 1379 de $1934^{8}$, comenzó a fortalecerse en el país la enseñanza profesional de los idiomas extranjeros ${ }^{9}$, toda vez que su currículo también comprendía idiomas modernos, lo que en gran medida ayudó a generar estrategias para la inclusión de estos en los planes de estudios de las instituciones educativas colombianas.

En 1979, a partir de una alianza internacional, se instauró la enseñanza obligatoria del inglés en los grados 6 y 7 , y del francés para grados 10 y $11^{10}$. Más adelante, con el fin de mejorar la enseñanza y el aprendizaje de los idiomas, se propuso el "Syllabus de inglés" desarrollado por el MEN en colaboración con el Consejo Británico y el Centro Colombo Americano; la propuesta buscaba principalmente guiar los objetivos en los colegios, renovar las estrategias de enseñanza y aprendizaje y ayudar en la búsqueda de material y textos actualizados para la enseñanza del idioma. Sin embargo, el proyecto no tuvo el impacto esperado debido a la falta de dominio del idioma por parte de los docentes y a las pocas condiciones para su aplicación en las instituciones escolares.

Otro paso importante para la enseñanza de los idiomas en Colombia fue el proyecto cofe (Colombian Framework for English), implementado para los programas de formación de docentes en algunas universidades del país entre 1991 y 1996. Este programa tuvo como principales objetivos ${ }^{11}$

6 Las políticas lingüísticas y las agendas de reformas que preceden al Programa Nacional en Colombia se remontan a los tiempos de la Colonia. Después de la colonización del "nuevo" continente los misioneros católicos fueron efectivos en imponer sus idiomas, principalmente el español, el griego y el latín. Más tarde, después de que la región se independizó de España, hace dos siglos, la nueva élite gobernante comenzó a enviar a sus hijos a Europa, lo que luego condujo a la importación de libros e ideas relacionados con lenguas tales como el francés, el alemán y el inglés. Estos movimientos facilitaron el camino de estas lenguas hacia el país y su asociación con ideas ilustradas y élites intelectuales, mientras que las lenguas indígenas y criollas comenzaron a asociarse con la ignorancia y el subdesarrollo. (Traducción propia).

7 Helg, op. cit.

8 Ministerio de Educación Nacional de Colombia, “Decreto 1379 del 5 de julio de 1934". Diario oficial, año LXx, n. ${ }^{\circ} 22647,30$ de julio de 1934,6 .

9 Claudia Figueroa, “Orígenes, formación y proyección de las Facultades de Educación en Colombia 1930-1954", Revista Historia de la Educación Latinoamericana, 8 (2006): 208.

10 Usma Wilches, op cit.

11 Clara Inés Rubiano, Cristina Frodden y Gloria Cardona, "The Impact of the Colombian Framework for English (COFE) Project: An Insiders' perspective", Íkala, Revista de Lenguaje y Cultura, 5 (1-2), (2000): 37-54, https://aprendeenlinea.udea.edu.co/revistas/index. $\mathrm{php/ikala/article/view/8545.}$ 
To make a qualitative change in the initial training of teachers of English as a foreign language; to design and implement a permanent training program for the teachers of the entities linked to the Project, which would include both practical and theoretical components, and to establish appropriate evaluating procedures, to design and implement a permanent training Project for active teacher of grades 6-11 in the government sector, to include practical and theoretical components and to establish appropriate evaluating procedures, to set common standards for the teaching practice component in the initial training, to expand the scope of the project to the regions, involving regional universities, and, to develop existing regional resource centers, and to encourage the creation of others ${ }^{12}$.

COFE fue implementado por el Reino Unido y el Ministerio de Educación colombiano, pero también enfrentó dificultades, entre ellas las diferencias en las estructuras de las universidades, la limitante en los recursos, la falta de familiaridad de los docentes con la investigación, y el insuficiente liderazgo por parte de los coordinadores de los programas ${ }^{13}$, razones por las que no tuvo continuidad.

Por otro lado, en la Constitución Política de $1991^{14}$ se da "reconocimiento explícito de Colombia como una nación multilingüe y pluricultural", ratificado en los artículos 7 y 10 de la misma Constitución, donde "el Estado reconoce y protege la diversidad étnica y cultural de la nación colombiana", y se asume "El castellano (como) el idioma oficial de Colombia. Las lenguas y dialectos de los grupos étnicos son también oficiales en sus territorios. La enseñanza que se imparta en las comunidades con tradiciones lingüísticas propias será bilingüe”.

Más adelante, en 1994, a partir de la Ley General de Educación ${ }^{15}$ empieza a asumirse de una manera más institucional y oficial el énfasis del bilingüismo en los currículos escolares del país, planteando para básica primaria "la adquisición de elementos de conversación y de lectura al menos de una lengua extranjera", y para básica secundaria "la comprensión y capacidad de expresarse en una lengua extranjera", además de especificar como una de las áreas obligatorias y fundamentales la de "humanidades, lengua castellana e idiomas extranjeros".

De otra parte, debido al auge de la política de apertura económica del país que "desde el comienzo de los años noventa ha implicado por una parte un vuelco completo en las relaciones de Colombia con el resto del mundo y por otra, la valoración de las lenguas extranjeras, en particular el inglés como idioma

12 Realizar un cambio cualitativo en la formación inicial de los profesores de inglés como lengua extranjera; diseñar e implementar un programa de formación permanente para los docentes de las entidades vinculadas al proyecto, que incluyera ambos componentes prácticos y teóricos, y establecer procedimientos de evaluación apropiados; diseñar e implementar un proyecto de capacitación permanente para maestros activos de los grados 6-11 en el sector gubernamental, para incluir componentes prácticos y teóricos y establecer estándares comunes para el componente de práctica de enseñanza en la capacitación inicial, para ampliar el alcance del proyecto a las regiones con la participación de universidades regionales; desarrollar centros de recursos regionales existentes, y fomentar la creación de otros. (Traducción propia).

13 Usma Wilches, op. cit.

14 Constitución Política de Colombia, Consejo Superior de la Judicatura (cs)), Sala administrativa, Corte Constitucional (Bogotá: Imprenta Nacional, 2015), 14.

15 Ley 115 de febrero 8 de 1994 - Ley General de Educación, Ministerio de Educación Nacional - Colombia (Congreso de la República, 1994), 7, www.mineducacion.gov.co/1621/articles-85906_archivo_pdf.pdf. 
internacional”16, "hacia 1997 el Ministerio de Educación Nacional (MEN) anunció la apertura del 'Programa Nacional de Bilingüismo' destinado a expandir el conocimiento del inglés por parte de alumnos en el sector oficial del país" 17 .

Teniendo también como referencia la Ley General de Educación, desde "la concepción curricular centrada en procesos y competencias, de carácter flexible, participativo y abierto que propende por el desarrollo integral de las personas"18 y el énfasis de la enseñanza de los idiomas extranjeros como área obligatoria y fundamental desde la educación básica, en 1996 el MEN emite los "Lineamientos curriculares en idiomas extranjeros", caracterizándolos como

$<(\ldots)$ orientaciones pedagógicas para que los docentes del área se apropien de los elementos conceptuales básicos y hagan efectiva la autonomía para guiar los procesos, para atender las necesidades del diseño curricular dentro del proyecto educativo institucional, buscar oportunidades de manejo innovador en el área, y asumir y apropiarse de los avances científicos y tecnológicos

Entre tanto, dentro del Plan Decenal de Educación 2006-2016 ${ }^{19}$ se enfatizan los “Desafíos de la Educación en Colombia: los fines de la Educación y su Calidad en el siglo xxI", resaltando la globalización y la autonomía, proponiendo que desde el currículo se optara por "desarrollar competencias en lengua materna y por lo menos en una segunda lengua".

Así, la normatividad antes mencionada, los lineamientos curriculares, la apertura económica a los mercados extranjeros y el Plan Decenas, vinieron a conformar el punto de partida para el diseño e implementación del Programa Nacional de Bilingüismo 2004-2019 - Inglés como Lengua Extrajera: Una estrategia para la Competitividad ${ }^{20}$, el cual fue propuesto como uno de los Proyectos Estratégicos para la Competitividad (PEC), enfocado al "Mejoramiento de las competencias comunicativas en inglés como lengua extranjera en todos los sectores educativos" como estrategia para la implementación del bilingüismo en el país.

Otra base fundamental fue la planteada en la política de Estado Visión Colombia 201921, donde se postula el "incremento en la capacidad de los alumnos para comunicarse en una segunda lengua", base donde el mismo MEN hace énfasis en que "dominar una lengua extranjera representa una ventaja comparativa,

16 Anne-Marie Truscott de Mejía, Claudia Lucía Ordóñez y Laura Fonseca, "Lineamientos para la educación bilingüe en Colombia: Hacia una política coherente” (informe de investigación, Universidad de los Andes - Centro de investigación y formación en educación, julio de 2006), 6 .

17 Truscott de Mejía, Ordoñez y Fonseca, op. cit.

18 Ministerio de Educación Nacional - Colombia, “Lineamientos curriculares en idiomas extranjeros", 1996, 1, 2, www.mineducacion. gov.co/1759/articles-339975_recurso_7.pdf.

19 Ministerio de Educación Nacional - Colombia, “Plan Decenal de Educación 2006-2016: Pacto Social por la Educación”, 2006a, 17, 19, http://www.plandecenal.edu.co/cms/index.php/caja-de-herramientas

20 Ministerio de Educación Nacional - Colombia, "Programa Nacional de Bilingüismo 2004-2019 - Inglés como Lengua Extrajera: Una estrategia para la Competitividad” (ppt), 2004.

21 Departamento Nacional de Planeación - Colombia, "Visión Colombia 2019: II Centenario", https://www.dnp.gov.co/politicas-deestado/vision-colombia-2019/Paginas/programa-vision-colombia-2019.aspx. 
un atributo de su competencia y competitividad" 22 , requisito este que debe convertirse en una "competencia para todos". Lo anterior permitió ratificar la necesidad de fortalecer el "dominio del inglés como lengua extranjera" y que el MEN propusiera estrategias para el desarrollo de competencias comunicativas en ese idioma.

Finalmente, el 12 de julio de 2013 se gestó la Ley 1651, por medio de la cual se modificaron algunos artículos de la Ley 115 de 1994 y se dictaron otras Disposiciones-Ley de bilingüismo ${ }^{23}$. La misma presenta en detalle los requerimientos para el desarrollo de habilidades comunicativas en inglés, además de aclarar la ruta para la creación de programas en la enseñanza de los idiomas en el país.

\section{Claridades sobre bilingüismo}

Hablar de bilingüismo implica revisar algunos de los diferentes significados que se le han dado al término a través del tiempo; en ese sentido cabe aclarar, con Bermúdez y Fandiño que "es difícil determinar con exactitud una definición única ya que en las últimas décadas varios autores desde diferentes disciplinas han intentado precisar este concepto" ${ }^{24}$, además de que a lo largo del tiempo ha venido evolucionando dinámicamente.

Una de las concepciones más relevantes de bilingüismo es la de Bloomfield ${ }^{25}$ cuando afirma que bilingüe es quien becomes so proficient as to be indistinguishable from the native speakers around him ${ }^{26}$; por lo tanto bilingüismo en este caso es native control of two languages ${ }^{27}$ pero en algunos casos they carry over foreign peculiarities from their native languages ${ }^{28}$.

Soler ${ }^{29}$ y Bermúdez y Fandiño ${ }^{30}$ hacen un excelente trabajo recopilando algunas claridades sobre la evolución del término y su influencia para la definición de bilingüismo en Colombia. Es así como Soler ${ }^{31}$ da a conocer que "Haugen propone que [es] bilingüe aquel que utiliza expresiones completas y con significado en otra lengua" y que "Weinreich ha tomado una posición más neutral al definirlo como la práctica de utilizar dos lenguas alternativamente", para finalmente

\footnotetext{
22 Ministerio de Educación Nacional (MEN) - Colombia, “Programa Nacional de Bilingüismo 2004-2019..., op. cit.

23 Presidencia de la República (PRC) - Colombia, Ministerio de Educación Nacional, Ley 1651 de 2013.

24 Bermúdez y Fandiño, op. cit., 101.

25 Leonard Bloomfield, L, Language (Londres: George Allen \& Unwin Ltd., 1935), 55-56, https://es.scribd.com/document/6383057/ Bloomfield-Leonard-Language-1933.

26 ...en el caso extremo del aprendizaje de lenguas extranjeras se vuelve tan competente como para ser indistinguible de los hablantes nativos alrededor de él. (Traducción propia).

27 ...el control nativo de dos lenguas. (Traducción propia).

28 ...trasladan las peculiaridades extranjeras de sus lenguas nativas. (Traducción propia).

29 Sandra Soler Castillo, "Bilingüismo y actitudes lingüísticas de la comunidad indígena Inga ante el español y el inga", Cultura e Intercultura (2003): 1. http://www.ub.edu/filhis/culturele/inga.html

30 Bermúdez y Fandiño, op. cit.

31 Soler Castillo, op. cit.
} 
aclarar que "Wallace Lambert propone el término balanced bilingual, que se refiere a individuos perfectamente competentes en ambas lenguas".

Son varias las posturas conceptuales sobre bilingüismo, y de hecho Bermúdez y Fandiño ${ }^{32}$, en la tabla que llaman "Definiciones de bilingüismo", hacen un compendio de distintas descripciones del término:

- Para Bloomfield, el bilingüismo implica "un dominio de dos lenguas igual que un nativo". En otras palabras, es la capacidad para hablar dos lenguas como lo hacen los hablantes de lengua materna produciendo enunciados bien formados, portadores de significación.

- Según Haugen, la persona bilingüe puede utilizar expresiones completas y con significado en diferentes lenguas.

- Por su parte, Weinreich afirma que la práctica de utilizar dos lenguas de forma alternativa se denomina bilingüismo y las personas implicadas bilingües.

- En 1959, Weiss sostiene que el bilingüismo es el uso directo, activo y pasivo de dos lenguas por el mismo sujeto hablante; entendiendo por activo cuando se habla, y pasivo cuando se recibe o entiende.

- Macnamara define el bilingüismo como la capacidad de desarrollar algún nivel de competencia (hablar, leer, entender, escribir) en una segunda lengua.

- Para Mackey, el bilingüismo es la cualidad de una persona o de una población que hace uso corriente de dos lenguas sin una aptitud marcada por una lengua más que por la otra.

- Por su parte, Titone explica que el bilingüismo es la capacidad de un individuo de expresarse en una segunda lengua respetando los conceptos y las estructuras propias de la misma.

- En 1981, Blanco afirma que el bilingüe es aquella persona que es capaz de codificar y decodificar en cualquier grado, señales lingüísticas provenientes de dos idiomas diferentes.

- Según Cerdá Massó, el bilingüismo consiste en la aptitud del hablante para utilizar indistintamente dos lenguas. Por extensión, dícese de la condición sociolingüística por la que una comunidad emplea dos lenguas distintas para cubrir exactamente los mismos cometidos comunicativos colectivos y privados.

- Harding y Riley sostienen que los individuos que tienen la posibilidad de comunicarse en dos o más códigos lo hacen en contextos diferenciados que requieren usar uno u otro sistema lingüístico. Por ende, su vocabulario y su habilidad para hablar, escribir, escuchar o leer tiene distintos niveles de acuerdo con los usos que realiza en cada lengua.

- Para Romaine, el bilingüe es aquella persona que aprende un conjunto de significados con dos representaciones lingüísticas diferentes.

- Lam define bilingüismo como el fenómeno de competencia y comunicación en dos lenguas.

Finalmente, los mismos Bermúdez y Fandiño reflexionan sobre la forma como en las primeras definiciones de bilingüismo se enfatiza en el manejo perfecto de

32 Bermúdez y Fandiño, op. cit., 102. 
las dos lenguas, mientras que las más recientes hacen precisión en el desarrollo psicológico, la organización cognitiva y los niveles de comunicación, además de agregar el punto de apertura de la interrelación cultural, concepto relevante en esta visión investigativa. Por eso es importante revisar la caracterización del bilingüismo con base en la relación entre el lenguaje y el pensamiento, realizada por Bermúdez y Fandiño, toda vez que son piezas fundamentales para comprender la esencia del bilingüismo en Colombia. Plantean entonces el "bilingüismo balanceado y dominante", determinado desde el nivel de competencia lingüística desarrollado por una persona y clasificado desde el uso y la función de la lengua a partir de lo propuesto por Crystal, Hamers y Blanc y Alarcón. También presentan el "bilingüismo coordinado, compuesto y subordinado" desde las posturas de Weinreich, Paradis y Signoret; el coordinado, caracterizado por el dominio de las dos lenguas por parte del hablante como si fuera monolingüe (en ambas); el compuesto, que se presenta cuando el hablante necesita ambas lenguas para pensar y comunicarse, sin detectar las diferencias conceptuales y requiere los dos idiomas para comunicarse; mientras que el subordinado se da cuando una de las lenguas es afianzada como lengua materna y la otra está en desarrollo. Otros tipos de bilingüismo sustentados en Paradis son el simultáneo y el sucesivo, el primero, dado por el aprendizaje de dos lenguas al mismo tiempo y de manera paralela, mientras que el sucesivo se da después de contar con una primera lengua establecida o lengua materna. Finalmente, desde las propuestas de Lambert, Signoret y Vila, presentan el "bilingüismo aditivo y el sustractivo"; aditivo, caracterizado por la influencia cultural del aprendiente porque se considera que la segunda lengua le permite alcanzar un grado cultural, y el sustractivo porque el aprendiente considera que adquirir una segunda lengua lo lleva a un retroceso en el terreno cultural.

Particularmente en Colombia, desde el Programa Nacional de Bilingüismo y desde los Estándares Básicos de Competencias en Lenguas Extranjeras: Inglés, el término bilingüismo se aborda desde "los diferentes grados de dominio con los que un individuo logra comunicarse en más de una lengua y una cultura. Estos diversos grados dependen del contexto en el cual se desenvuelve cada persona"33; igualmente explicita que el manejo del inglés en el país tiene carácter de lengua extranjera toda vez que

(...) es aquella [lengua] que no se habla en el ambiente inmediato y local, pues las condiciones sociales cotidianas no requieren su uso permanente para la comunicación. Una lengua extranjera se puede aprender principalmente en el aula y, por lo general, el estudiante está expuesto al idioma durante períodos controlados. A pesar de no ser usada en circunstancias diferentes a las académicas, los estudiantes de una lengua extranjera pueden alcanzar altos niveles de desempeño para ser comunicadores eficientes cuando así lo requieran.

33 Ministerio de Educación Nacional - Colombia, "Estándares básicos de competencias en lenguas extranjeras: Inglés”, $2006 \mathrm{~b}, 5$. 
Así, se puede considerar que para la adquisición del inglés como lengua extranjera en Colombia el bilingüismo subordinado muestra fielmente los factores que se asumen en el país para la propuesta del área, dado que se cuenta con la adquisición de la lengua materna español mientras el inglés está en desarrollo; además del bilingüismo sucesivo, porque el aprendizaje del inglés se inicia después del dominio de la lengua materna. Por el contrario, la caracterización del bilingüismo aditivo y sustractivo en la educación colombiana no cuenta con la suficiente fundamentación para determinarlo, razón por la que se justifica realizar un estudio donde se identifique el impacto desde la cultura y la identidad (propias o foráneas) en las propuestas curriculares bilingües de las instituciones educativas de educación superior del país.

\section{Detalles del Programa Nacional de Bilingüismo en Educación Superior}

Después de algunas claridades históricas y conceptuales básicas sobre el bilingüismo, centrando la atención en Colombia, se analiza en detalle la estructura general del Programa Nacional de Bilingüismo (PNB) 2004-2019, específicamente los pasos dados desde el mismo en la Educación Superior del país, en especial desde las licenciaturas en idiomas / inglés ofertadas a escala regional en las universidades colombianas, población objeto del proceso investigativo doctoral.

El PNB 2004-2019 cuenta con estrategias y proyectos encaminados a "lograr ciudadanos y ciudadanas capaces de comunicarse en inglés, de tal forma que puedan insertar al país en los procesos de comunicación universal, en la economía global y en la apertura cultural, con estándares internacionalmente comparables" ${ }^{34}$, habiendo determinado previamente "las metas de nivel de desempeño en el idioma a través de las diferentes etapas del proceso educativo". Para suplir esa necesidad se escogió el Marco Común Europeo de Referencia para Lenguas: Aprendizaje, Enseñanza y Evaluación (MCER), elaborado por el Consejo Europeo y adoptado por el MEN para organizar la "Guía 22" o "Estándares Básicos de Competencias en Lenguas Extranjeras", y determinar la propuesta general del proceso de bilingüismo en el país.

Las metas específicas del PNB están centradas en tres grandes poblaciones: en primer lugar, estuvo la Educación Básica y Media, donde se propone que los estudiantes de grado 11 egresen con un nivel intermedio (B1) desde el MCER, además de que los docentes de esta primera población cuenten con un nivel mínimo de B2. En segundo lugar, la Educación Superior, donde se propone que los futuros profesores de inglés egresen con un nivel $\mathrm{B} 2+\mathrm{o} \mathrm{C} 1$, y los profesionales de otras carreras cuenten con un nivel intermedio de B2. La última población corresponde a los institutos de idiomas, determinando en la ley ${ }^{35}$ que los programas deben ser registrados y acreditados con base en la normatividad establecida para ser ofertados.

34 Ibíd., 6.

35 Presidencia de la República, Ministerio de Educación, Ley 1651 del 12 de julio de 2013. 
Para la Educación Básica se contó inicialmente con la formulación, publicación y socialización de los "Estándares Básicos de Competencias en Lenguas Extranjeras", además del fortalecimiento de los niveles de lengua y las competencias metodológicas de los docentes en servicio del país, estrategia liderada por el MEN y desarrollada con el apoyo de las secretarías de educación certificadas $^{36}$. Para los institutos de idiomas, regidos inicialmente por el Decreto 4904 de 2009 y la Norma Técnica Colombiana 5580, se formalizó el registro y la acreditación de la oferta de programas mediante la Ley 1651 de 2013.

En Educación Superior se contó con varias estrategias, entre las que se destaca el Proyecto de Fortalecimiento a Licenciaturas en Idiomas / Inglés ${ }^{37}$. Para el desarrollo de este proyecto se convocaron pares amigos, quienes lo lideraron teniendo como objetivos principales realizar un diagnóstico general de la situación académica de los programas y proponer un plan de mejoramiento para fortalecer las licenciaturas en idiomas de las universidades en Colombia.

Igualmente, para realizar el proyecto se establecieron tres fases: una fase diagnóstica inicial, recolectando información con diferentes instrumentos y analizándola con el fin de establecer las fortalezas y los puntos a mejorar en las licenciaturas en idiomas; los instrumentos utilizados fueron entrevistas, encuestas, grupos focales, exámenes, observación de clases y visitas a la planta física. Una segunda fase de formulación, compromisos y acompañamiento, que permitió llegar a acuerdos durante las visitas de los pares amigos formulando un plan de mejoramiento de los programas participantes, a ser implementado por la Institución de Educación Superior (IES) con el acompañamiento del MEN en la tercera y última fase.

Cuando se indaga sobre el impacto de los proyectos del PNB implementados por el Ministerio de Educación Nacional, se encuentra que son múltiples las revisiones, que incluyen análisis de política desde la inclusión, la exclusión y la estratificación ${ }^{38}$; análisis crítico del discurso de los Estándares en Inglés ${ }^{39}$; análisis de la política a nivel central - Bogotá ${ }^{40}{ }^{41}$; avances sobre el estudio del bilingüismo en Colombia ${ }^{42}$; impacto del bilingüismo en currículos de educación básica y media ${ }^{43}{ }^{44}$; estudios económicos regionales en el nivel de bachillerato ${ }^{45}$;

36 Ministerio de Educación Nacional - Colombia, "Programa Nacional de Bilingüismo 2004-2019...," op. cit., 8.

37 Ministerio de Educación Nacional - Colombia, "Proyecto de fortalecimiento a licenciaturas en idiomas / Inglés", ppt, 2007.

38 Usma Wilches, op. cit.

39 Carmen Helena Guerrero, "Colombia bilingüe: ¿Qué significa ser bilingüe en el marco del Plan Nacional de Bilingüismo?, Profile: Issues in Teachers' Professional Development, 10, n. ${ }^{\circ}$ 1, (2008): 27-46.

40 María Claudia Moreno Marulanda, "Análisis de la implementación de las políticas públicas de bilingüismo en Bogotá" (trabajo de grado de maestría, Universidad de La Salle, Facultad de Educación, 2009).

41 Adriana Esmeralda Angarita Trujillo y Carmen Aura Arias Castilla, "Aproximación a los antecedentes del bilingüismo en Colombia y la formación de educadores bilingües", Horizontes pedagógicos, 12, n. ${ }^{\circ} 1$ (2010).

42 Bermúdez y Fandiño, op. cit., 99-124.

43 Claudia Lucía Ordóñez, "Educación para el bilingüismo en contexto monolingüe: dos lenguas conectadas desde el currículo", Estudios de Lingüistica Inglesa Aplicada (ELIA), n. ${ }^{\circ} 10$ (2010): 41-76.

44 Truscott de Mejía, Ordóñez y Fonseca, op. cit.

45 Andrés Sánchez Java, "El bilingüismo en los bachilleres colombianos", Documentos de trabajo sobre economía regional - Banco de la República, n. ${ }^{\circ} 159$ (2012). 
evaluación nacional de políticas en educación en términos de acceso, equidad, calidad y pertinencia ${ }^{46}$; multidimensionalidad del bilingüismo ${ }^{47}$; implicaciones para el $\mathrm{PNB}^{48}{ }^{49}$; retos del $\mathrm{PNB}^{50}$; estudio cuantitativo del bilingüismo en Colombia ${ }^{51}$; balance intermedio de la implementación del $\mathrm{PNB}^{52}$; proyección de la enseñanza del inglés a través del método AICLE en Colombia ${ }^{53}$; bilingüismo con enfoque en etnoeducación ${ }^{54}$; desafíos del bilingüismo en Educación Superior con base en la experiencia en una universidad colombiana ${ }^{55}$; la investigación como fundamento para el diseño curricular de una licenciatura en inglés como lengua extranjera ${ }^{56}$.

Indiscutiblemente también se debe revisar el aspecto cultural que interviene en el aprendizaje y la enseñanza de una lengua extranjera, al respecto se perfilan temas como definiciones de cultura y su estrecha relación con la identidad ${ }^{57} 58$ 59 , identidad cultural y educación en Paulo Freire ${ }^{60}$, estudios de movimientos sociales, enmarcamientos y cultura ${ }^{61}$, definiciones de cultura, cultura e identidad $^{62}$, particularidades y generalidades de la cultura y el lenguaje ${ }^{63}$, aspectos psicológicos y de comportamiento en la cultura ${ }^{64}{ }^{65}$, críticas por la

46 OECD/International Bank for Reconstruction and Development/The World Bank, Reviews of National Policies for Education: Tertiary Education in Colombia 2012, oECD Publishing, http://dx.doi.org/ 10.1787/9789264180697-en.

47 Ferney Cruz Arcila, "La multidimensionalidad del bilingüismo: consideraciones conceptuales e implicaciones en torno al Plan Nacional de Bilingüismo", Revista de la Universidad de La Salle, n. 59 (2012).

48 José David Herazo, Sonia Jerez y Danilza Lorduy, "Opportunity and incentive for becoming bilingual in Colombia: Implications for Programa Nacional de Bilingüismo", Íkala, Revista de Lenguaje y Cultura, 17, n. ${ }^{\circ} 2$ (mayo-agosto 2012): 199-213.

49 Anne-Marie Truscott de Mejía, "Bilingual Education in Colombia: Towards a Recognition of Languages, Cultures, and Identities", Colombian Applied Linguistics Journal, n. ${ }^{\circ} 8$ (2006): 152-168.

50 Yamith José Fandiño-Parra, Jenny Raquel Bermúdez-Jiménez y Víctor Elías Lugo-Vásquez, "Retos del Programa Nacional de Bilingüismo. Colombia Bilingüe", Educación y Educadores, 15, n. ${ }^{\circ}$ 3, (2012): 363-381.

51 Andrés Sánchez Java, "El bilingüismo en Colombia”, Documentos de trabajo sobre economía regional - Banco de la República, n. ${ }^{\circ} 191$ (2013).

52 Rosalba Cárdenas y Norbella Miranda, "Implementación del Programa Nacional de Bilingüismo en Colombia: un balance intermedio", Educación y Educadores, 17, n. ${ }^{\circ}$ 1, (2014): 51-67.

53 John Morales Osorio, Luis Cabas Vásquez y Carlos Vargas Mercado, "Projection of teaching of bilingualism through AICLE method in Colombia”, Revista Lasallista de Investigación, 14, n. ${ }^{\circ} 1$ (2017): 84-92.

54 Margoth Guzmán Munar, "Bilingüismo y etnoeducación en Colombia”, Cuadernos de Lingüística Hispánica, n. 7 (2006): $75-84$.

55 Carlo Granados Beltrán, "Desafíos del bilingüismo en Educación Superior: La experiencia del Departamento de Lenguas de la Universidad Central en Bogotá, Colombia”, GIST: Education \& Learning Research Journal, 7, (2013): 245-258.

56 Nélida Cárdenas Vergaño, "La investigación como fundamento para el diseño curricular de la Licenciatura en inglés como lengua extranjera", Revista de Investigaciones UNAD, 13, n. ${ }^{\circ} 1$ (junio de 2014), http://dx.doi.org/10.22490/25391887.1135.

57 E. B. Tylor, Primitive Culture: Researches into the Development of Mythology, Philosophy, Religion, Language, Art and Custom. (1873), London: John Murray.

58 R. Rohner, "Toward a conception of culture for cross-cultural psychology". Journal of Cross-Cultural Psychology, vol. 15, (1874): $11-38$.

59 R. Linton, The Cultural Background of Personality. (1945): New York: Appleton-Century.

60 Norman Estupiñan Quiñones y Nubia Agudelo Cely, "Identidad Cultural y Educación en Paulo Freire: Reflexiones en torno a estos conceptos". Revista Historia de la Educación Latinoamericana, vol. 10, (2012): 25-40.

61 Luis Fernando Villafuerte Valdés. "Una metodología interpretativa para el estudio de los movimientos sociales. Enmarcamientos y cultura. Una visión desde México”. Revista Historia de la Educación Latinoamericana, vol. 11, (2008): 225-246.

62 Patricio Guerrero A. "Antropología y cultura. Una mirada crítica a la identidad, diversidad, alteridad y diferencia”. Quito: Abya-Yala, 2002, ISBN: 9978-22-247-2.

63 Josiane, Hamers y Michel, Blanc. "Social psychological aspects of bilinguality: culture and identity". Bilinguality and Bilingualism. Chapter 8, (2011): 198-24. Online ISBN: 9780511605796.

64 M. H Segall, J. W. Berry, P. R. Dasen y Y. H. Poortinga, Human Behavior in the Global Perspective: An Introduction to Cross-Cultural Psychology. (1990), New York: Pergamon Press.

65 J. S. Bruner, Acts of Meaning. (1990), Cambridge, MA and London: Harvard University Press. 
uniformidad en el uso de la lengua y la implantación de una nueva cultura en los pueblos $^{66}$, identidad cultural y globalización ${ }^{67}$, la necesidad del fortalecimiento de la identidad cultural en la educación superior ${ }^{68}$ y de abordar las diversas dimensiones del aprendizaje de las lenguas para fortalecer el bilingüismo ${ }^{69}$, además de muchas otras, que seguirán documentando y fortaleciendo la propuesta investigativa doctoral.

\title{
4. Propuesta investigativa doctoral: "Políticas bilingües y su impacto en la identidad cultural y calidad educativa en programas de licenciatura en len- guas - inglés en Colombia, 2004-2014"
}

En 2012, la Organización para la Cooperación y el Desarrollo Económicos (OCDE) y el Banco Mundial presentaron el informe de Evaluaciones de Políticas Nacionales de Educación, caso Educación Superior en Colombia ${ }^{70}$, donde explicitan que no hay documentos que evalúen la eficacia o el impacto del PNB:

\begin{abstract}
El gobierno colombiano ha seguido el camino correcto al considerar que las medidas para la adquisición del dominio de un segundo idioma deben aplicarse a todo el sistema educativo con especial hincapié en las etapas de educación primaria y secundaria. Como se ha demostrado en otros países, es inviable que las instituciones de educación superior asuman la responsabilidad total en el proceso de adquisición de un segundo idioma. Sin embargo, los miembros del equipo evaluador no tuvieron conocimiento de dato alguno que demostrase la eficacia de la iniciativa bilingüe colombiana, siete años después de su puesta en marcha (238).
\end{abstract}

Lo anterior, más la revisión y reflexión citadas en los anteriores apartados sobre los estudios alrededor del bilingüismo en Colombia, permite plantear una propuesta investigativa doctoral que determine el impacto del Proyecto de Fortalecimiento a Programas de Licenciatura en Lenguas - Inglés (PFPLI) en los currículos de las licenciaturas en inglés de tres universidades regionales colombianas en relación con la calidad educativa y la identidad cultural.

Esta investigación educativa particularmente busca: reconocer los principales aspectos históricos y culturales sobre la implementación del bilingüismo en Colombia; caracterizar las propuestas curriculares en tres universidades regionales del país, determinando el impacto del PFPLI en el fortalecimiento de la

66 Fandiño Parra et al, op cit.

67 J. Dias Sobrinho, "Compromiso Social de la Educación Superior". Hacia una política regional de aseguramiento de la calidad de la educación superior para América Latina y el Caribe. Ana Lúcia Gazzola y Sueli Pires (coord.). (2008): Instituto Internacional de la UNESCO para la Educación Superior en América Latina y el Caribe UNESCO-IESALC.

68 Francisco López Segrera, "Impacto del Marco de Acción Prioritaria para el Cambio y Desarrollo de la Educación Superior". La educación superior en América Latina y el Caribe: diez años después de la Conferencia Mundial de 1998. (2008): Colombia: Pontificia Universidad Javeriana - UNESCO.

69 Silvia Valencia Giraldo, "Bilingualism and English language teaching in Colombia: A critical outlook". (2005): ELT conference. Universidad del Quindío, Armenia. October.

70 OCDE/International Bank for Reconstruction and Development/The World Bank, op. cit. 
identidad cultural de sus programas ${ }^{71}$; determinar la eficacia del PFPLI analizando los resultados de las pruebas Saber Pro (ECAES) en el periodo comprendido entre 2004 y 2014; y establecer la pertinencia y relevancia del PFPLi para las licenciaturas en idiomas en el ámbito regional. Se plantea entonces una perspectiva mixta de investigación ${ }^{72}$ dado que el método permite al investigador combinar datos cuantitativos(informaciónestadística)condatoscualitativos(historiasoexperencias personales), dándole fortaleza a la investigación y permitiendo una mejor comprensión del problema; además, se asume un enfoque hermenéutico crítico toda vez que la información siempre estará sujeta a diferentes interpretaciones, por eso es indispensable contar no solo con esta de manera física sino también realizar una revisión de los estudios, comentarios y otras interpretaciones a los que ha dado lugar. Esto permite que el investigador interprete, argumente, capte y critique desde diferentes contextos, ampliando el significado de la información y generando un proceso innovador y creativo que enriquece el tema tanto desde su descripción comprensiva como desde una visión crítica y social ${ }^{73}$ que permitirá comprender e interpretar la situación particular para determinar de una manera crítica $^{74}$ el impacto del Proyecto de Fortalecimiento a Programas de Licenciatura en Lenguas - Inglés (PFPLI) del Ministerio de Educación Nacional.

De la misma manera, en este proceso investigativo se asume lo propuesto por Stake $^{75}$ (1995) en cuanto a que es estudio de caso colectivo seleccionado por el investigador con un número de casos coyunturales para examinar fenómenos o poblaciones con condiciones generales similares, permitiendo seleccionar instituciones, poblaciones o actores determinados en función de la investigación (o su tema particular), visualizando las complejidades presentes y concluyendo deductivamente, en vista de que se realizarán descripciones completas y apropiadas con un enfoque crítico de la información encontrada ${ }^{76}$. Se definió una muestra de carácter heterogéneo que permita dilucidar relaciones entre variables y que garantice unidades suficientes de un determinado tipo ${ }^{77}$, al igual que esté orientada por la información inherente a la investigación en la medida que permita maximizar la utilidad de esa información desde muestras particulares seleccionadas con base en la expectativa del contenido ${ }^{78}$.

Por lo tanto, se asumen como principales características de selección de la muestra del proceso investigativo: 1 . Universidades con un programa de Licenciatura en Idiomas que haya participado en las tres fases del PFPLI del MEN

71 María Concepción Barrón Tirado, "Análisis de contenidos curriculares. Un acercamiento metodológico, en Procesos y didácticas de la formación universitaria, coord. por E. Chehaybar y R. Amador (México: UnAM, Centro de Estudios sobre la Universidad, 2003), 75-96.

72 John W. Creswell, A Concise Introduction to Mixed Methods Research (U.S.A.: University of Nebraska-Lincoln/saGe Publications, 2015).

73 Mauricio Martínez, Comportamiento humano. Nuevos métodos de investigación (México: Editorial Trillas, 1999).

74 E. Guba \& Y. Lincoln, "What is Constructivist Paradigm Anyway?", en Fourth Generation Evaluation (sage Publications, 1989).

75 R. Stake, The art of Case Study Research (California: SAGe Publications, 1995).

76 G. Neiman y G. Quaranta, "Los estudios de caso en la investigación sociológica”, en Estrategias de investigación cualitativa, comp. por Vasilachis de Gialdino (Buenos Aires: Gedisa, 2006).

77 J. Padua, et al. Técnicas de investigación aplicadas a las ciencias sociales (Chile: Fondo de Cultura Económica, 1994).

78 B. Flyuberg, "Five Misunderstandings about Case Study Research", Qualitative Inquiry, 12:2 (California: SAGE Publications, 2006). 
y haya renovado registro curricular entre el periodo 2004-2014; esto con el fin de establecer parámetros comparativos de las mallas curriculares desde el antes y el después de la aplicación del Proyecto de Fortalecimiento. 2. Que las universidades por investigar pertenezcan a la Red de Universidades Estatales de Colombia (Rudecolombia), con el fin de aunar esfuerzos de recursos humanos y económicos para "comprender, teorizar e intervenir los factores de las culturas socioeducativas, desde la perspectiva del trabajo en red en el contexto internacional y la construcción de nación desde la región", y así "consolidar la formación de investigadores críticos y solidarios en el campo de la educación desde líneas y grupos de investigación del mismo programa doctoral"79. 3. Que los programas por investigar pertenezcan a universidades regionales para profundizar en los aspectos culturales y de identidad que se tienen en cuenta para el aprendizaje y la enseñanza de un idioma extranjero, además de consolidar la investigación respecto a la problemática educativa regional colombiana. 4 . Finalmente, pero no menos importante, que haya interés de parte de las instituciones de Educación Superior en participar en la investigación, garantizándose el acceso a la información requerida desde la revisión documental y la aplicación de instrumentos a la población seleccionada.

Esta investigación permitirá reconocer, identificar, analizar y dar una perspectiva crítica de la forma como las políticas educativas nacionales han tenido o no en cuenta las necesidades y particularidades culturales y curriculares regionales para su formulación y ejecución. De otro lado, también permitirá presentar una visión prospectiva sobre los aspectos curriculares de calidad educativa e identidad cultural que deberían plantearse al formular políticas o proyectos de bilingüismo en el marco regional en instituciones de educación superior.

\section{FUENTES}

Ministerio de Educación Nacional - Colombia. “Plan Decenal de Educación 2006-2016: Pacto Social por la Educación", 2006a, 17, 19. http://www.plandecenal.edu.co/cms/index.php/caja-de-herramientas.

Ministerio de Educación Nacional - Colombia. “Estándares Básicos de Competencias en Lenguas Extranjeras: Inglés", 2006b.

Ministerio de Educación Nacional - Colombia. “Programa Nacional de Bilingüismo 2004-2019 - Inglés como Lengua Extrajera: Una estrategia para la Competitividad" (ppt), 2004.

Ministerio de Educación Nacional - Colombia. "Lineamientos Curriculares en Idiomas Extranjeros". 1996, 1, 2. www.mineducacion.gov.co/1759/articles-339975_recurso_7.pdf

Ministerio de Educación Nacional. Ley 115 de febrero 8 de 1994 - Ley General de Educación. (Congreso de la República de Colombia - CRC, 1994), 7. www.mineducacion.gov.co/1621/articles-85906_archivo_pdf.pdf

Ministerio de Educación Nacional de Colombia. Decreto 1379 del 5 de julio de 1934. Diario oficial. Año Lxx, n. ${ }^{\circ}$ 22647, 30 de julio de 1934, 6. http://www.suin-juriscol.gov.co/viewDocument. asp?id=1739954

79 Centro Virtual de Noticias de la Educación, Red de Universidades Estatales de Colombia (Rudecolombia), 2010, http://www. mineducacion.gov.co/cvn/1665/w3-article-220396.html. 


\section{REFERENCIAS}

Beltrán, Carlo Granados. “Desafíos del bilingüismo en Educación Superior: La experiencia del Departamento de Lenguas de la Universidad Central en Bogotá, Colombia". gIST: Education E Learning Research Journal, 7 (2013): 245-258.

Bermúdez, Jenny Raquel y Yamith José Fandiño. “El fenómeno bilingüe: perspectivas y tendencias en bilingüismo". Revista de la Universidad de La Salle, n. 59 (2012): 99-124.

Bloomfield, Leonard. Language. Londres: George Allen \& Unwin Ltd., 1935.

Bruner, J. S. Acts of Meaning. (1990): Cambridge, MA and London: Harvard University Press.

Cárdenas, Rosalba y Norbella Miranda. "Implementación del Programa Nacional de Bilingüismo en Colombia: un balance intermedio". Educación y Educadores, 17, n. ${ }^{\circ} 1$ (2014): 51-67.

Cárdenas Vergaño, Nélida. "La investigación como fundamento para el diseño curricular de la Licenciatura en inglés como lengua extranjera". Revista de Investigaciones UNAD, 13, n. ${ }^{\circ} 1$ (junio de 2014), http://dx.doi.org/10.22490/25391887.1135.

Castillo Trujillo, Adriana Esmeralda y Carmen Aura Arias Castilla. "Aproximación a los antecedentes del bilingüismo en Colombia y la formación de educadores bilingües". Horizontes pedagógicos, $12, \mathrm{n}^{\circ} 1$ (2010).

Centro Virtual de Noticias de la Educación. Red de Universidades Estatales de Colombia (Rudecolombia). 2010. http://www.mineducacion.gov.co/cvn/1665/w3-article-220396.html

Constitución Política de Colombia. Consejo Superior de la Judicatura (csj), Sala Administrativa, Corte Constitucional. Bogotá: Imprenta Nacional, 2015.

Creswell, John W. A concise introduction to mixed methods research. U.S.A.: University of Nebraska-Lincoln/sage Publications, 2015.

Cruz Arcila, Ferney. "La multidimensionalidad del bilingüismo: consideraciones conceptuales e implicaciones en torno al Plan Nacional de Bilingüismo". Revista Universidad de La Salle, n. ${ }^{\circ}$ 59 (2012).

Departamento Nacional de Planeación. "Visión Colombia 2019: II Centenario". https://www.dnp. gov.co/politicas-de-estado/vision-colombia-2019/Paginas/programa-vision-colombia-2019. aspx.

Dias Sobrinho, J. “Compromiso Social de la Educación Superior". Hacia una política regional de aseguramiento de la calidad de la educación superior para América Latina y el Caribe. Ana Lúcia Gazzola y Sueli Pires (coord.), (2008): Instituto Internacional de la UNESCO para la Educación Superior en América Latina y el Caribe UNESCO-IESALC.

Estupiñan Quiñones, Norman y Agudelo Cely, Nubia. “Identidad Cultural y Educación en Paulo Freire: Reflexiones en torno a estos conceptos". Revista Historia de la Educación Latinoamericana, vol. 10, (2012): 25-40.

Fandiño-Parra, Yamith José, Bermúdez-Jiménez, Jenny Raquel y Lugo-Vásquez, Víctor Elías. “Retos del Programa Nacional de Bilingüismo. Colombia Bilingüe". Educación y Educadores, 15, n. ${ }^{\circ}$ 3, (2012): 363-381.

Figueroa, Claudia. “Orígenes, formación y proyección de las Facultades de Educación en Colombia 1930-1954". Revista Historia de la Educación Latinoamericana, 8 (2006): 208.

Flyuberg, B. "Five Misunderstandings about Case Study Research", Qualitative Inquiry, 12:2 (2006).

Guba, E. \& Lincoln, I. "What is Constructivist Paradigm Anyway?". En Fourth Generation Evaluation, Newbury Park: Sage Publications (1989).

Guerrero, Carmen Helena. “Colombia bilingüe: ¿Qué significa ser bilingüe en el marco del Plan Nacional de Bilingüismo? Profile: Issues in Teachers' Professional Development, 10, n. ${ }^{\circ}$ 1, (2008): 27-46.

Guerrero A, Patricio. "Antropología y cultura. Una mirada crítica a la identidad, diversidad, alteridad y diferencia". Quito: Abya-Yala, 2002, ISBN: 9978-22-247-2.

Guzmán Munar, Margoth. "Bilingüismo y etnoeducación en Colombia". Cuadernos de Lingüística Hispánica, n. ${ }^{\circ} 7$ (2006): 75-84.

Hamers Josiane y Blanc Michel. "Social psychological aspects of bilinguality: culture and identity". Bilinguality and Bilingualism. Chapter 8, (2011): 198-24. Online ISBN: 9780511605796.

Helg, Aline. La educación en Colombia, 1918-1957: Una historia social, económica y política. Bogotá: Uni- 
versidad Pedagógica Nacional / Plaza y Janés Editores, 2001.

Herazo, José David, Sonia Jerez y Danilza Lorduy. "Opportunity and incentive for becoming bilingual in Colombia: Implications for Programa Nacional de Bilingüismo", Íkala, Revista de Lenguaje y Cultura, 17, n. ${ }^{\circ}$ (2012): 199-213.

Linton R., The Cultural Background of Personality. (1945), New York: Appleton-Century.

López Segrera, Francisco. "Impacto del Marco de Acción Prioritaria para el Cambio y Desarrollo de la Educación Superior". La educación superior en América Latina y el Caribe: diez años después de la Conferencia Mundial de 1998. (2008): Colombia: Pontificia Universidad Javeriana - UNESCO.

Martínez, Mauricio. Comportamiento humano. Nuevos métodos de investigación. 2. a ed. México: Editorial Trillas, 1999.

Morales Osorio, John, Luis Cabas Vásquez y Carlos Vargas Mercado. "Projection of teaching of bilingualism through AICLE method in Colombia". Revista Lasallista de Investigación, 14, n. $^{\circ} 1$ (2017): 84-92. 10.22507/rli.v14n1a7.

Moreno Marulanda, María Claudia. “Análisis de la implementación de las políticas públicas de Bilingüismo en Bogotá". Trabajo de grado para maestría. Universidad de La Salle, Facultad de Ciencias de la Educación, 2009.

Neiman G. y G. Quaranta. "Los estudios de caso en la investigación sociológica". En Estrategias de investigación cualitativa, compilado por Vasilachis de Gialdino. Buenos Aires: Gedisa, 2006.

Ordóñez, Claudia Lucía. "Educación para el bilingüismo en contexto monolingüe: dos lenguas conectadas desde el currículo". Estudios de Lingüística Inglesa Aplicada (eLIA), n. ${ }^{\circ} 10$ (2010): 41-76.

OECD/International Bank for Reconstruction and Development/The World Bank, Reviews of National Policies for Education: Tertiary Education in Colombia 2012, oECD Publishing. http:// dx.doi.org/ 10.1787/9789264180697-en.

Padua, Jorge, Ingvar Ahman, Héctor Apezechea y Carlos Borsotti. Técnicas de investigación aplicadas a las ciencias sociales. Chile: Fondo de Cultura Económica, 1994.

Presidencia de la República - Colombia (PRC). Ley 1651 de 2013. Bogotá: Ministerio de Educación Nacional, 2013.

Rohner R., "Toward a conception of culture for cross-cultural psychology". Journal of Cross-Cultural Psychology, vol. 15, (1874): 11-38.

Rubiano, Clara Inés, Cristina Frodden y Gloria Cardona. "The Impact of the Colombian Framework for English (COFE) Project: An Insiders' perspective". Íkala: Revista de Lenguaje y Cultura, 5, n. ${ }^{\circ}$ 1-2 (2000): 37-54. https://aprendeenlinea.udea.edu.co/revistas/index.php/ikala/article/ view/8545

Sánchez Java, Andrés. "El bilingüismo en los bachilleres colombianos". Documentos de trabajo sobre economía regional - Banco de la República, n. ${ }^{\circ} 159$ (2012).

Sánchez Java, Andrés. "El bilingüismo en Colombia". Documentos de trabajo sobre economía regional Banco de la República, n. ${ }^{\circ} 191$ (2013).

Segall, M. H, Berry, J. W., Dasen, P. R. y Poortinga, Y. H. Human Behavior in the Global Perspective: An Introduction to Cross-Cultural Psychology. (1990), New York: Pergamon Press.

Soler Castillo, Sandra. "Bilingüismo y actitudes lingüísticas de la comunidad indígena Inga ante el español y el inga". Cultura e Intercultura (2003): 1. http://www.ub.edu/filhis/culturele/inga. html

Stake, Robert E. The art of Case Study Research. California: Sage, 1995.

Truscott de Mejía, Anne-Marie, Claudia Lucía Ordóñez y Laura Fonseca. “Lineamientos para la educación bilingüe en Colombia: Hacia una política coherente". Informe de investigación, Universidad de los Andes - Centro de investigación y formación en educación, julio de 2006.

Truscott de Mejía, Anne-Marie. "Bilingual Education in Colombia: Towards a Recognition of Languages, Cultures, and Identities". Colombian Applied Linguistics Journal, n. 8 (2006): 152-168.

Tylor, E. B., Primitive Culture: Researches into the Development of Mythology, Philosophy, Religion, Language, Art and Custom. (1873): London: John Murray.

Usma Wilches, Jaime A. "Education and Language Policy in Colombia: Exploring Processes of Inclusion, Exclusion, and Stratification in Times of Global Reform". Profile: Issues in Teachers' 
Professional Development, 11, n. ${ }^{\circ} 1$ (2009): 2.

Valencia Giraldo, Silvia. "Bilingualism and English language teaching in Colombia: A critical outlook". (2005): ELT conference. Universidad del Quindío, Armenia. October.

Villafuerte Valdés, Luis Fernando. "Una metodología interpretativa para el estudio de los movimientos sociales. Enmarcamientos y cultura. Una visión desde México". Revista Historia de la Educación Latinoamericana, vol. 11, (2008): 225-246.

Como citar:

Cárdenas Vergaño, Nélida. “Perspectivas para un estudio sobre bilingüismo en universidades regionales colombianas" Revista Historia de la Educación Latinoamericana. Vol. 20 No. 31 (2018): 125-142 DOI: 10.19053/01227238.8566 